\section{BINTRAFUSP ALFA IN COMBINATION WITH CHEMOTHERAPY IN PATIENTS WITH STAGE IV NSCLC: SAFETY AND PHARMACOKINETIC RESULTS OF THE INTR@PID LUNG 024 STUDY}

${ }^{1}$ Christian Rolfo*, ${ }^{2}$ Laurent Greillier, ${ }^{3}$ Remi Veillon, ${ }^{4}$ Firas Badin, ${ }^{5}$ Francois Ghiringhelli, ${ }^{6}$ Nicolas Isambert, ${ }^{7}$ Astrid Paulus, ${ }^{8}$ Marc Lambrechts, ${ }^{9}$ Surendra Chaudhary, ${ }^{9}$ Xiaoli You, ${ }^{9}$ Yulia Vugmeyster, ${ }^{10}$ Christoph Helwig, ${ }^{11}$ Sandrine Hiret. ${ }^{1}$ Mount Sinai Health System, New York, NY, USA, New York, NY, USA; ${ }^{2}$ Aix Marseille University, Assistance Publique-Hôpitaux de Marseille, Marseille, France, Marseille, France; ${ }^{3}$ Centre Hospitalier Universitaire de Bordeaux, Bordeaux, France, Bordeaux, France; ${ }^{4}$ Baptist Health Lexington, Lexington, KY, USA, Lexington, KY, USA; ${ }^{5}$ Centre Georges François Lederc, Dijon, France, Dijon, France; ${ }^{6} \mathrm{CHU}$ de Poitiers, Poitiers, France, Dijon Cedex, France; ${ }^{7} \mathrm{CHU}$ Sart Tilman, Liege, Belgium, Liege, Belgium; ${ }^{8}$ Algemeen Ziekenhuis Sint-Maarten, Mechelen, Belgium, Mechelen, Belgium; ${ }^{9}$ EMD Serono, Billerica, MA USA, Billerica, MA, USA; ${ }^{10}$ The healthcare business of Merck KGaA, Darmstadt, Germany, Darmstadt, Germany; ${ }^{11}$ Institut de Cancérologie de I'Ouest, Saint-Herblain, France, Saint Herblain, France

Background Bintrafusp alfa is a first-in-class bifunctional fusion protein composed of the extracellular domain of the TGF- $\beta$ RII receptor (a TGF- $\beta$ "trap") fused to a human IgG1 mAb blocking PD-L1. Here we report cumulative safety and pharmacokinetic (PK) results from the global, phase $1 \mathrm{~b} / 2$ INTR@PID LUNG 024 study (NCT03840915), which evaluated bintrafusp alfa in combination with chemotherapy (CT) in patients with stage IV NSCLC.

Methods Adult patients with stage IV nonsquamous or squamous NSCLC and an ECOG PS $\leq 1$ were included. Cohorts $\mathrm{A}, \mathrm{B}$, and $\mathrm{C}$ included patients with no prior systemic therapy; patients in cohort $\mathrm{D}$ had disease that progressed with previous anti-PD-(L)1 therapy. Cohorts received bintrafusp alfa 2400 mg every 3 weeks intravenously in combination with CT for 4 cycles (A [nonsquamous only]: cisplatin or carboplatin + pemetrexed; B: carboplatin + nab-paclitaxel or paclitaxel; C: cisplatin or carboplatin + gemcitabine; D: docetaxel) followed by bintrafusp alfa maintenance (monotherapy or in combination with pemetrexed in cohort A) for up to 31 cycles. The primary objective of this study was to evaluate the safety of bintrafusp alfa in combination with CT. Dose-limiting toxicities (DLTs) were assessed during a 3 -week observation period. Serial samples were drawn to assess serum concentration and calculate PK parameters by noncompartmental analysis.

Results As of the May 5, 2021, data cutoff, 70 patients received bintrafusp alfa in combination with CT. Of 35 patients included in the DLT analysis, 4 experienced 1 DLT according to a safety monitoring committee (data cutoff May $5,2021 ; A: n=1 / 8 ; B: n=1 / 8 ; C: n=0 / 8 ; D: n=2 / 11)$. Cumulative safety data are reported in table 1 . PK data were available for 67 patients (A: $n=38$; B: $n=9 ; C: n=8$; $: n=12$ ). PK profiles were similar across cohorts and between patients who did and did not experience a DLT. Observed bintrafusp alfa first-cycle exposures (Cmax, AUC, and Ctrough) were consistent with the published population PK (popPK) model. ${ }^{1}$
Abstract 465 Table 1 Safety results from the INTR@PID LUNG 024 study

\begin{tabular}{|c|c|c|c|c|}
\hline & $\begin{array}{c}\text { Cohort A } \\
(\mathrm{n}=40)\end{array}$ & $\begin{array}{c}\text { Cohort B } \\
(n=9)\end{array}$ & $\begin{array}{c}\text { Cohort C } \\
(n=9)\end{array}$ & $\begin{array}{l}\text { Cohort D } \\
(\mathrm{n}=12)\end{array}$ \\
\hline $\mathrm{DLTs}, \mathrm{n} / \mathrm{N}^{*}(\%)$ & $1 / 8(12.5)$ & $1 / 8(12.5)$ & $0 / 8(0)$ & $2 / 11(18.2)$ \\
\hline \multicolumn{5}{|l|}{ TEAEs, $n(\%)$} \\
\hline Any & $40(100.0)$ & $9(100.0)$ & $9(100.0)$ & $12(100.0)$ \\
\hline Grade $\geq 3$ & $32(80.0)$ & $8(88.9)$ & $7(77.8)$ & $12(100.0)$ \\
\hline Grade $\geq 4$ & $16(40.0)$ & $3(33.3)$ & $5(55.6)$ & $6(50.0)$ \\
\hline Leading to dose reduction of CT & $14(35.0)$ & $3(33.3)$ & $5(55.6)$ & $4(33.3)$ \\
\hline $\begin{array}{l}\text { Leading to permanent discontinuation } \\
\text { of bintrafusp alfa }\end{array}$ & $16(40.0)$ & $1(11.1)$ & $5(55.6)$ & $4(33.3)$ \\
\hline $\begin{array}{l}\text { Leading to permanent discontinuation } \\
\text { of } \mathrm{CT}\end{array}$ & $16(40.0)$ & $1(11.1)$ & $4(44.4)$ & $3(25.0)$ \\
\hline \multicolumn{5}{|l|}{ Treatment-related AEs, $\mathbf{n}(\%)$} \\
\hline Any bintrafusp alfa-related AEs & $35(87.5)$ & $9(100.0)$ & $9(100.0)$ & $12(100.0)$ \\
\hline Bintrafusp alfa-related serious AEs & $12(30.0)$ & $1(11.1)$ & $1(11.1)$ & $3(25.0)$ \\
\hline $\begin{array}{l}\text { Bintrafusp alfa-related AEs leading to } \\
\text { death }\end{array}$ & 0 & 0 & 0 & 0 \\
\hline \multicolumn{5}{|l|}{ AEs of special interest, $n(\%)$} \\
\hline Any skin lesions ${ }^{\dagger}$ & $6(15.0)$ & $1(11.1)$ & $4(44.4)$ & $1(8.3)$ \\
\hline Any immune-related AEs & $19(47.5)$ & $3(33.3)$ & $5(55.6)$ & $6(50.0)$ \\
\hline Any infusion-related reactions & $5(12.5)$ & $1(11.1)$ & $1(11.1)$ & $1(8.3)$ \\
\hline Anemia & $20(50.0)$ & $6(66.7)$ & $9(100.0)$ & $9(75.0)$ \\
\hline Any bleeding events & $19(47.5)$ & & $8(88.9)$ & $8(66.7)$ \\
\hline \multicolumn{5}{|c|}{$\begin{array}{l}\text { AE, adverse event; CT, chemotherapy; DLT, dose-limiting toxicity; TEAE, treatment-emergent adverse } \\
\text { event. } \\
\text { *Assessed in patients in the safety part of the study. } \\
\text { tDefined as actinic keratosis, basal cell carcinoma, Bowen's disease, hyperkeratosis, } \\
\text { keratoacanthoma, lip squamous cell carcinoma, and squamous cell carcinoma of skin. Actinic } \\
\text { keratosis, hyperkeratosis, and keratoacanthoma were reported in this study. }\end{array}$} \\
\hline
\end{tabular}

Conclusions The safety profile of bintrafusp alfa in combination with CT was manageable and similar to that reported for ICIs in combination with CT, with the exception of TGF- $\beta$ related skin lesions known to occur with TGF- $\beta$ inhibition. No new safety signals were identified and there were no treatment-related deaths. The PK profile was consistent with the predicted monotherapy popPK model, suggesting no victim DDI potential for bintrafusp alfa with CT.

Acknowledgements The authors thank the patients and their families, investigators, co-investigators, and the study teams at each of the participating centers, at the healthcare business of Merck KGaA, Darmstadt, Germany, and at EMD Serono, Billerica, Massachusetts, USA.

Trial Registration NCT03840915

\section{REFERENCE}

1. Wilkins JJ, Vugmeyster $Y$, Dussault I. Population pharmacokinetic analysis of bintrafusp alfa in different cancer types. Adv Ther 2019;36:2414-2433.

Ethics Approval The trial was approved by each site's independent ethics committee.

http://dx.doi.org/10.1136/jitc-2021-SITC2021.465 